\title{
Nanoscale
}

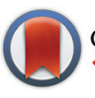

CrossMark \& click for updates

Cite this: Nanoscale, 2014, 6, 12655

\section{Fine structural tuning of whereabout and clustering of metal-metal oxide heterostructure for optimal photocatalytic enhancement and stability $\dagger$}

\author{
Minmin Gao, ${ }^{a}$ Connor Kang Nuo Peh, ${ }^{\mathrm{a}}$ Yanlin Pan, ${ }^{\mathrm{b}}$ Qing-Hua Xu ${ }^{\mathrm{b}}$ and \\ Ghim Wei Ho*a,c
}

\begin{abstract}
In this work, we demonstrate the fine structural tuning of metal-metal oxide heterostructure with regards to the individual tuning of the various core and shell components from shell thickness to metal core constitution. Furthermore, we deliberately engineered spatially confined and clustered Au nanoparticles in the core of a porous shell structure without the assistance of template or linker. Our findings unambiguously highlight that whilst it is important to incorporate metal nanoparticles into metal oxide for higher photocatalytic performance through enhanced light absorption and charge separation, the "whereabout" and clustering of Au nanoparticles affect the photocatalytic performance. Furthermore, we also prove the enhanced and prolonged catalytic activity of spatially confined metal cores over conventional surface loaded metal particles, which originates from the structural stability and optimized contact interface for heterojunction-induced charge transfer. The present well-controlled synthetic route can offer a facile and valuable way to tune and probe specific structure in relation to nanoscale light-matter manipulation and solar-to-chemical energy conversion studies.
\end{abstract}

Received 18th June 2014

Accepted 6th August 2014

DOI: $10.1039 / c 4 n r 03373 f$

www.rsc.org/nanoscale

\section{Introduction}

The development of photocatalysts with porous morphologies mainly aims to increase the specific surface area and decrease the migration distance of charge carrier, while innovation in the hetero-interfacing of photocatalyst with metal nanoparticles aims to enhance light absorption, charge separation and transfer efficiencies. The combination of these strategies can provide a powerful approach to rationally design novel material that boost light energy conversion systems such as solar cell, photoelectrochemical, photocatalysis for water splitting and environmental remediation.

In the context of hetero-interfacing, various metals with metal oxide systems have been developed. ${ }^{1-5}$ In several cases, the metal particles are simply loaded onto the surfaces of the semiconductors as isolated island to produce limited heterointerfaces, while more uncommonly reported is a spatial confinement of metal nanoparticle within metal oxide matrix. The confinement of the nanoparticles as core with the metal oxide

\footnotetext{
${ }^{a}$ Department of Electrical and Computer Engineering, National University of Singapore, 4 Engineering Drive 3, Singapore 117576, Singapore.E-mail: elehgw@nus.edu.sg

${ }^{b}$ Department of Chemistry, National University of Singapore, 3 Science Drive 3, Singapore 117543, Singapore

${ }^{c}$ Institute of Materials Research and Engineering, ${ }^{*}$ STAR (Agency for Science,

Technology and Research), 3 Research Link, Singapore 117602, Singapore

$\dagger$ Electronic supplementary information (ESI) available. See DOI: 10.1039/c4nr03373f
}

shell protects and isolates the nanoparticles from leaching and coalescence during catalytic reactions. In addition, the coreshell architecture provides strong interaction between the metal core and shell that may boost catalytic activity. Furthermore, the utilization of the spatial confinement of metal nanoparticle for photochemical studies is limited. Hence, some fundamental questions that need to be address i.e. "whereabouts" coupling of metal nanoparticles and how the "clustering" of metal nanoparticles may affect photocatalytic properties.

There are various chemical synthesis methods of fabricating spatially confined metal particles in metal oxide system i.e. yolk-shell, eccentric and concentric core-shell, which include layer-by-layer deposition and sacrificial templating techniques. ${ }^{6,7}$ Nevertheless, it is highly challenging to design and synthesize such heterostructure because of formidable difficulties in controlling its growth kinetics to achieve specific structure. It is noted that a well-controlled synthetic route can facilitate the probing of specific structure to elucidate the influence of various structural components on photochemical properties. It is also noteworthy that most of the reported synthetic processes involve the use of additional materials as a support to form the shell or a linker between the core and shell. ${ }^{8-11}$ This is often followed by etching process to remove the supporting material and/or introduce porosity to the shell. Such multistep procedures reduce controllability in terms of metal nanoparticle core sizes and dispersivity, as well as shell porosity and crystallinity. Moreover, using additional materials 
to encapsulate metal nanoparticles may decrease the accessibility of the reactants to the active sites. Furthermore, calcination is generally carried out to convert $\mathrm{TiO}_{2}$ from amorphous to crystalline phase or for the precipitation of metal nanoparticles, which will cause the detrimental effect of metal nanoparticles sintering leading to a loss in reactivity. Therefore, the design of highly controllable template or capping ligand free synthesis method to fabricate the core-shell heterostructure of appropriate porosity and structural stability is desirable.

Herein, we have rationally designed a metal-metal oxide heterostructure catalyst with spatially confined metal particles, attaining porous core-shell structure. The synthesis is simple and tunable to independently control, various core and shell structure components from shell thickness to single or multi cores constitution. In addition to our synthetic effort, we have systematically evaluated the unique features afforded by the core-shell morphology based on various model systems for photocatalytic understanding. We demonstrated that the "whereabout" and clustering of Au nanoparticles matter considerably to photocatalytic performance. Furthermore, we have proven the enhanced and prolonged catalytic activity of spatially confined metal cores over conventional surface loaded metal particles, which originates from the structural stability and optimized heterojunction-induced charge transfer attributes.

\section{Results and discussion}

The schematic of Fig. 1a shows the structural tuning of the four different designs of $\mathrm{TiO}_{2}$ nanospheres synthesized with/ without Au nanoparticles, as well as surface loaded or spatially confined $\mathrm{Au}$ nanoparticles. The samples were synthesized such as to study the effects of Au nanoparticles "whereabouts" and core constitution on photocatalytic properties. In the context of $\mathrm{TiO}_{2}$ nanostructure synthesis, hydrofluoric acid (HF) has been reported as a structure directing agent to attain better morphological control. ${ }^{12}$ However, in this work, $\mathrm{TiF}_{4}$ precursor is used in place of HF to avoid the direct handling of hazardous acid because the hydrolysis of $\mathrm{TiF}_{4}$ during hydrothermal reaction promotes the in situ release of HF. The introduction of $\mathrm{TiF}_{4}$ also preferentially exposes the $\{001\}$ facet of anatase $\mathrm{TiO}_{2}$, which based on theoretical calculations, has enhanced the reactivity because of higher concentration of low coordination $\mathrm{Ti}_{5 \mathrm{c}}$ centers. ${ }^{12,13}$

Fig. $1 \mathrm{~b}$ and inset show the SEM image of $\mathrm{TiO}_{2}$ nanospheres with a rough spherical morphology of an average diameter $\sim 250 \mathrm{~nm}$. The TEM image (Fig. $\mathrm{S} 1 \dagger$ ) shows that the $\mathrm{TiO}_{2}$ nanospheres are made up of randomly shaped $\mathrm{TiO}_{2}$ nanocrystals, which have self-assembled into a spherical shape. The sizes of the $\mathrm{TiO}_{2}$ nanospheres can be controlled via the amount of $\mathrm{TiF}_{4}$ added. In addition, different nanospheres sizes ranging from diameter of $200 \mathrm{~nm}$ to $1 \mu \mathrm{m}$ were obtained with increasing $\mathrm{TiF}_{4}$ (Fig. S2 $\dagger$ ). Next, Au on $\mathrm{TiO}_{2}$ or surface loaded Au nanoparticles was synthesized through a physical blending process. The uniform loading of $\mathrm{Au}$ nanoparticles onto $\mathrm{TiO}_{2}$ nanospheres surface can be seen from Fig. 1c and inset. On the other hand, Au@ $\mathrm{TiO}_{2}$, or spatially confined Au nanoparticles, was prepared through the hydrothermal treatment of $\mathrm{TiF}_{4}$ and $\mathrm{Au}$ nanoparticles mixture to promote the formation of coresshell structures (Fig. 1d and e). A series of experiments have proven that both the amounts of $\mathrm{TiF}_{4}$ and $\mathrm{Au}$ nanoparticles affect the shell thickness and the multiplicity of the cores in
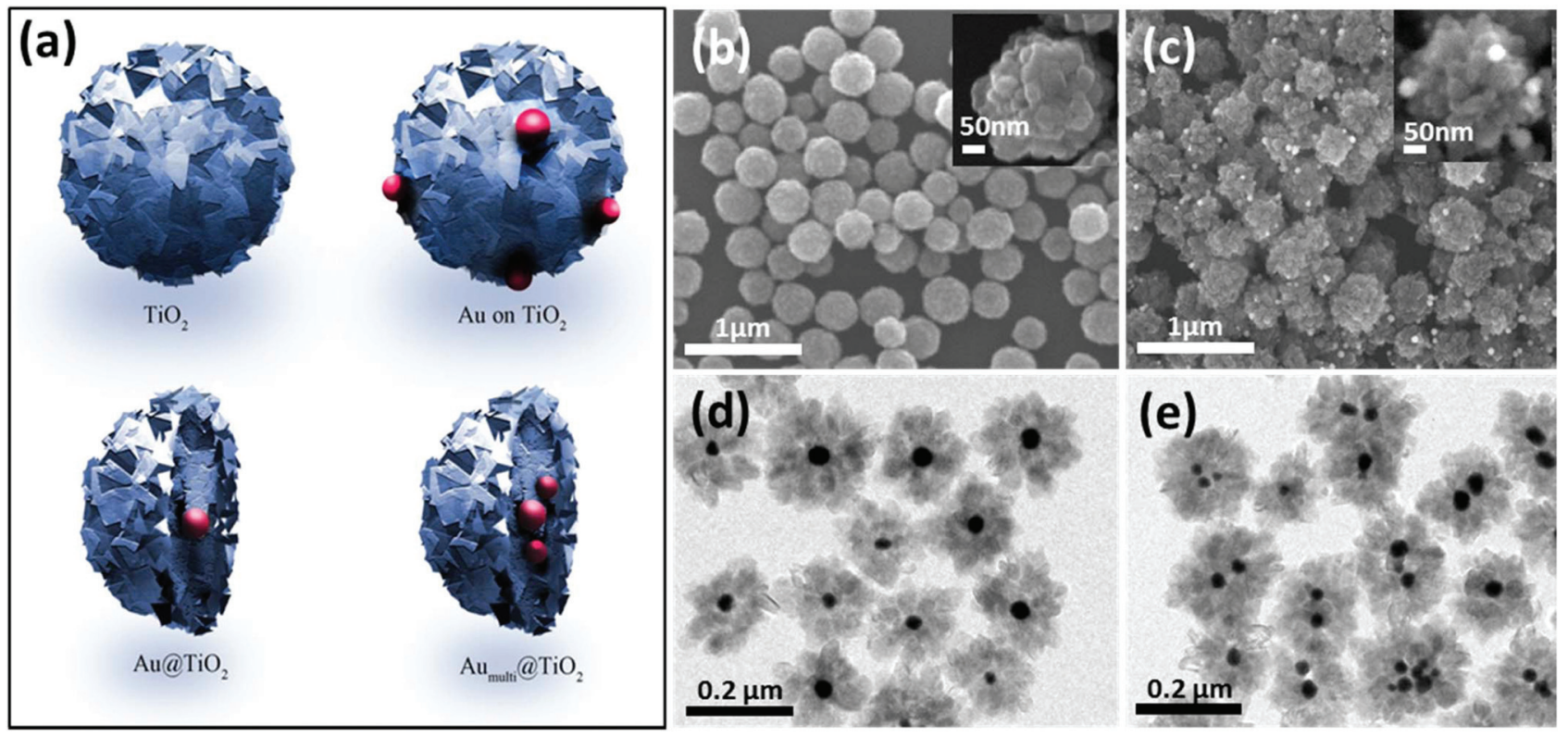

Fig. 1 (a) Schematic of the four different designs of $\mathrm{TiO}_{2}$ nanospheres synthesized with/without Au nanoparticles, as well as surface loaded or spatially confined Au nanoparticles. SEM images of (b) $\mathrm{TiO}_{2}$ nanospheres and (c) $\mathrm{Au}$ on $\mathrm{TiO}_{2}$ nanospheres or surface loaded Au nanoparticles on $\mathrm{TiO}_{2}$ nanospheres. Insets show high magnification of the respective samples. (d-e) TEM images of Au@TiO ${ }_{2}$ or spatially confined Au nanoparticles of single and mostly multiple cores. 
$\mathrm{Au} @ \mathrm{TiO}_{2}$ (the amounts of the reagents are expressed as volume ratios (\%) of the stock solutions). At lower $\mathrm{TiF}_{4}$ volume $(<12 \%)$, the increase in $\mathrm{Au}$ nanoparticles volume ratio effectively decreases the $\mathrm{Au@TiO}{ }_{2}$ shell thickness (Fig. S2†). The average shell thicknesses (particle sizes) are $\sim 115 \mathrm{~nm}$ (250 nm), $90 \mathrm{~nm}(200 \mathrm{~nm}), 65 \mathrm{~nm}(150 \mathrm{~nm})$ and $40 \mathrm{~nm}$ $(100 \mathrm{~nm})$ with the $\mathrm{Au}$ nanoparticles concentrations of $5 \%$, $10 \%, 20 \%$, and $30 \%$ respectively (Fig. $\mathrm{S} 2 \dagger$ ). At higher $\mathrm{TiF}_{4}$ concentrations $(>12 \%)$, the increase in the $\mathrm{Au}$ nanoparticles induced the formation of multiple $\mathrm{Au} @ \mathrm{TiO}_{2}$ structures (Fig. S2 $\uparrow$ ). However, when the Au nanoparticles concentrations are too low $(<10 \%), \mathrm{TiO}_{2}$ nanospheres without $\mathrm{Au}$ cores were observed amongst the single and multiple $\mathrm{Au} @ \mathrm{TiO}_{2}$. It is noted that when the $\mathrm{TiF}_{4}$ and $\mathrm{Au}$ nanoparticle concentrations were very high ( $20 \%$ and $40 \%$ respectively), some of the nanospheres were observed to have fused, which resulted in $\mathrm{Au}$ nanoparticles core clustering (Fig. S2 $\dagger$ ). The fusing was likely because of the condensation reaction of the surface hydroxyl groups of two adjacent $\mathrm{Au} @ \mathrm{TiO}_{2}$ nanospheres. Here, the employed hydrothermal synthesis was demonstrated to be facile and controllable such that the independent tuning of various core and shell structure components from shell thickness to single or multi cores constitution can be demonstrated.

The time-dependent morphological evolution of $\mathrm{Au} @ \mathrm{TiO}_{2}$ was studied by taking snapshots at different reaction times. At an initial reaction stage of $<15 \mathrm{~min}, \mathrm{TiF}_{4}$ hydrolysates and $\mathrm{Au}$ nanoparticles mixture were observed (Fig. S3a-b $\dagger$ ). After 30-45 $\mathrm{min}, \mathrm{TiF}_{4}$ hydrolysates were deposited on the Au nanoparticles surface without well-defined structure (Fig. S3c-d $\dagger$ ).
The supersaturated system results in the formation of the individual $\mathrm{TiO}_{2}$ nanocrystals observed in the shell structure. Because the surface of $\mathrm{Au}$ nanoparticle acts as preferential (heterogeneous) nucleation sites for $\mathrm{TiO}_{2}$, the core-shell nanostructures are preferentially formed when $\mathrm{Au}$ nanoparticles are present. On increasing the reaction time to $1 \mathrm{~h}$, individual core-shell nanospheres were formed (Fig. S3e †). The amount of fluoride ions released at this stage promoted the preferential growth of low-energy (101) $\mathrm{TiO}_{2}$ facets on $\mathrm{Au}$ nanoparticles, which produced nanospheres with relatively sharp and tapered tips. As the $\mathrm{TiF}_{4}$ hydrolysis continued (Fig. S3f $\dagger$ ), the nucleation and growth of $\mathrm{TiO}_{2}$ species onto the tapered tips evolved into nanospheres with cropped/flattened tips. In addition to the prolong reaction time, the high concentration of $\mathrm{F}^{-}$ionterminated (004) crystal planes produced a cropped-tip morphology. ${ }^{14}$ The morphology transformation can be attributed to its surface chemistry, in which adsorbate ions impose significant effects on the relative stability of different $\mathrm{TiO}_{2}$ crystal planes.

A detailed chemical analysis was carried out using EDX mapping to study the elemental composition and distribution throughout the Au@TiO ${ }_{2}$ core-shell nanospheres. The images in Fig. 2a-d correspond to the obtained bright field image, $\mathrm{O}$ K-edge, Ti K-edge and Au M-edge respectively. It can be seen that the $\mathrm{O}$ and $\mathrm{Ti}$ signals are found throughout the nanosphere, while the Au signal is located in the center part of the nanosphere. A line scan of a single nanosphere was also performed as plotted in Fig. 2e, which correlates to the elemental mapping of $\mathrm{Au}$ and $\mathrm{TiO}_{2}$ with core-shell structure. The size distribution of $\mathrm{Au}$ nanoparticles that was used to confine the
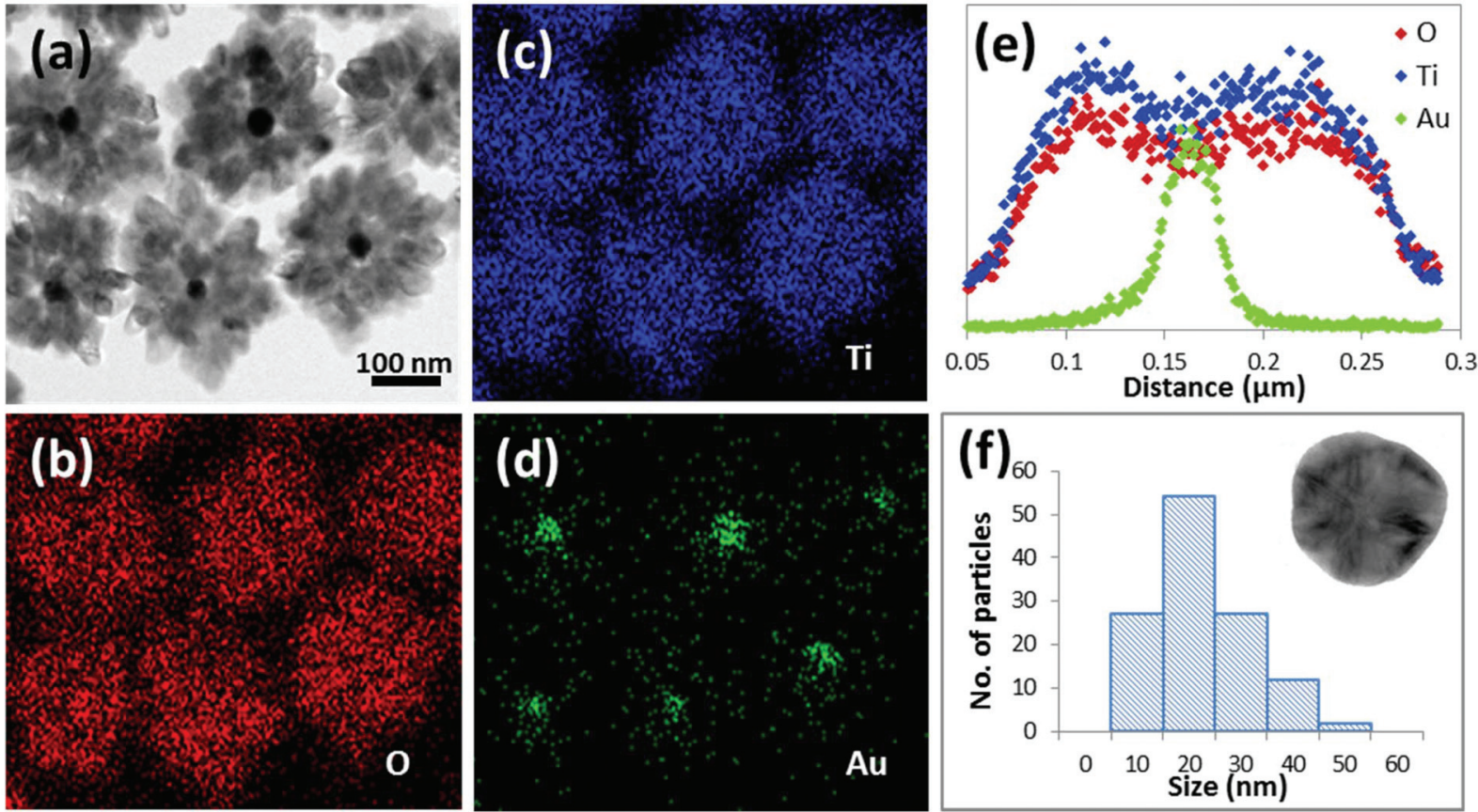

Fig. 2 (a-d) TEM image of the single core $\mathrm{Au}_{\mathrm{aTiO}}$ and its respective STEM EDX elemental mapping images. (e) Cross-sectional compositional line profiles of a single AuCTiO . (f) Histogram of the size distribution of Au nanoparticles. Inset image as-synthesized multi-twinned polyhedron Au nanoparticle. 
$\mathrm{TiO}_{2}$ nanospheres is shown in Fig. 2f. The Gaussian distribution shown in the histogram indicates the size distribution of Au nanoparticles with size 10-50 nm. The Au cores are generally quasi-spherical particles, or more specifically are of multi-twinned polyhedrons (Fig. 2f inset). The quasi spherical shape of the Au cores is found to be well-preserved in the assynthesized core-shell nanospheres.

The TEM image shown in Fig. 3a indicates the various locations where high resolution imaging is carried out. It is observed from Fig. $3 \mathrm{~b}$ that a good interface and continuity exist between $\mathrm{Au}$ nanoparticle and $\mathrm{TiO}_{2}$ shell. This suggests a close confinement of the $\mathrm{Au}$ nanoparticles within the $\mathrm{TiO}_{2}$ matrix, thus maximizing the contact area between the interfaces. The light contrast in between the $\mathrm{TiO}_{2}$ nanoparticles indicates the presence of porosity within the shell. The highmagnification image of the $\mathrm{TiO}_{2}$ shell (Fig. 3c) shows the adjacent lattice spacing of $0.35 \mathrm{~nm}$, attributed to the (101) lattice planes of the $\mathrm{TiO}_{2}$ anatase. The high resolution image of $\mathrm{Au}$ core (Fig. 3d) shows the lattice fringes of 0.242 and $0.202 \mathrm{~nm}$, which correspond to (111) and (200) of face-centered cubic metallic Au respectively. ${ }^{16}$ The XRD spectra (Fig. 3e) of all the nanospheres have shown to possess highly crystalline anatase phase without undergoing any post annealing treatment. The $\mathrm{TiO}_{2}$ anatase diffraction peaks (101), (004), (200), (105), (211) and (204) are observed at approximately $2 \theta$ of $25.3^{\circ}, 37.8^{\circ}$, $48.1^{\circ}, 53.9^{\circ}, 55.1^{\circ}$ and 62.8 (JCPDS no. 21-1272), respectively. As compared to the pristine $\mathrm{TiO}_{2}$ nanospheres, the main anatase diffraction peaks of $\mathrm{Au} @ \mathrm{TiO}_{2}$ (single and multiple cores) samples were sharper and stronger, indicating the formation of strong electronic interaction between $\mathrm{Au}$ nanoclusters and $\mathrm{TiO}_{2} \cdot{ }^{15}$ Furthermore, the Au@TiO 2 spectra for both single and multiple cores exhibit additional diffraction peaks at $38.1^{\circ}, 44.3^{\circ}$ and $64.5^{\circ}$, which can be assigned to the (111), (200) and (220) planes of Au nanoparticles (JCPDS 4-0783). The core-shell nanospheres assume a non-alloy structure as individual $\mathrm{Au}$ and $\mathrm{TiO}_{2}$ diffraction peaks can be obtained. The $\mathrm{N}_{2}$ adsorption-desorption isotherm and the pore size distribution curves (Fig. 3f) indicate that the $\mathrm{Au} @ \mathrm{TiO}_{2}$ is present as mesoporous structures with narrow pore size distributions centered mainly at $12 \mathrm{~nm}$. On the basis of the calculations based on desorption branches using the Brunauer-Emmett-Teller and Barrett-Joyner-Halenda model, the surface area of the Au@TiO ${ }_{2}$ is determined to be $38.5 \mathrm{~m}^{2} \mathrm{~g}^{-1}$. The BET analysis shows that the Au@ $\mathrm{TiO}_{2}$ core-shell nanospheres consist of porous shell that strongly favors accessibility to active interfacial sites on Au core. Here, it is concluded that the assynthesized confined $\mathrm{Au}$ cores with porous $\mathrm{TiO}_{2}$ shells offer passage for water, as well as the satisfactory isolation of nanoparticles against agglomeration.

We have systematically tailored various core and shell components from shell thickness, to core multiplicity in order to evaluate their effects on photocatalytic $\mathrm{H}_{2}$ production. Fig. 4a shows the photocatalytic $\mathrm{H}_{2}$ production of different shell thickness with fixed $20 \mathrm{~nm}$ Au core. Here, an average $\mathrm{H}_{2}$ evolution of $188 \mu \mathrm{mol} \mathrm{h}^{-1} \mathrm{~g}^{-1}$ is observed with the shell thicknesses of 65-140 nm (particle sizes between 150-300 nm). However, further increase in shell thickness to 190 and $390 \mathrm{~nm}$ (particle size of 400 and $800 \mathrm{~nm}$ ) decreases the average $\mathrm{H}_{2}$ evolution drastically to 162 and $51 \mu \mathrm{mol} \mathrm{h} \mathrm{h}^{-1} \mathrm{~g}^{-1}$ respectively. The decrease in photocatalytic reactivity is likely because of a decrease in the composition ratio of $\mathrm{Au}$ to $\mathrm{TiO}_{2}$ as the shell thickness increases. Next, the synthesis of the core-shell structures was fixed at a shell thickness of $\sim 90 \mathrm{~nm}$ equivalent to particle size of diameter $200 \mathrm{~nm}$. The absorption spectra
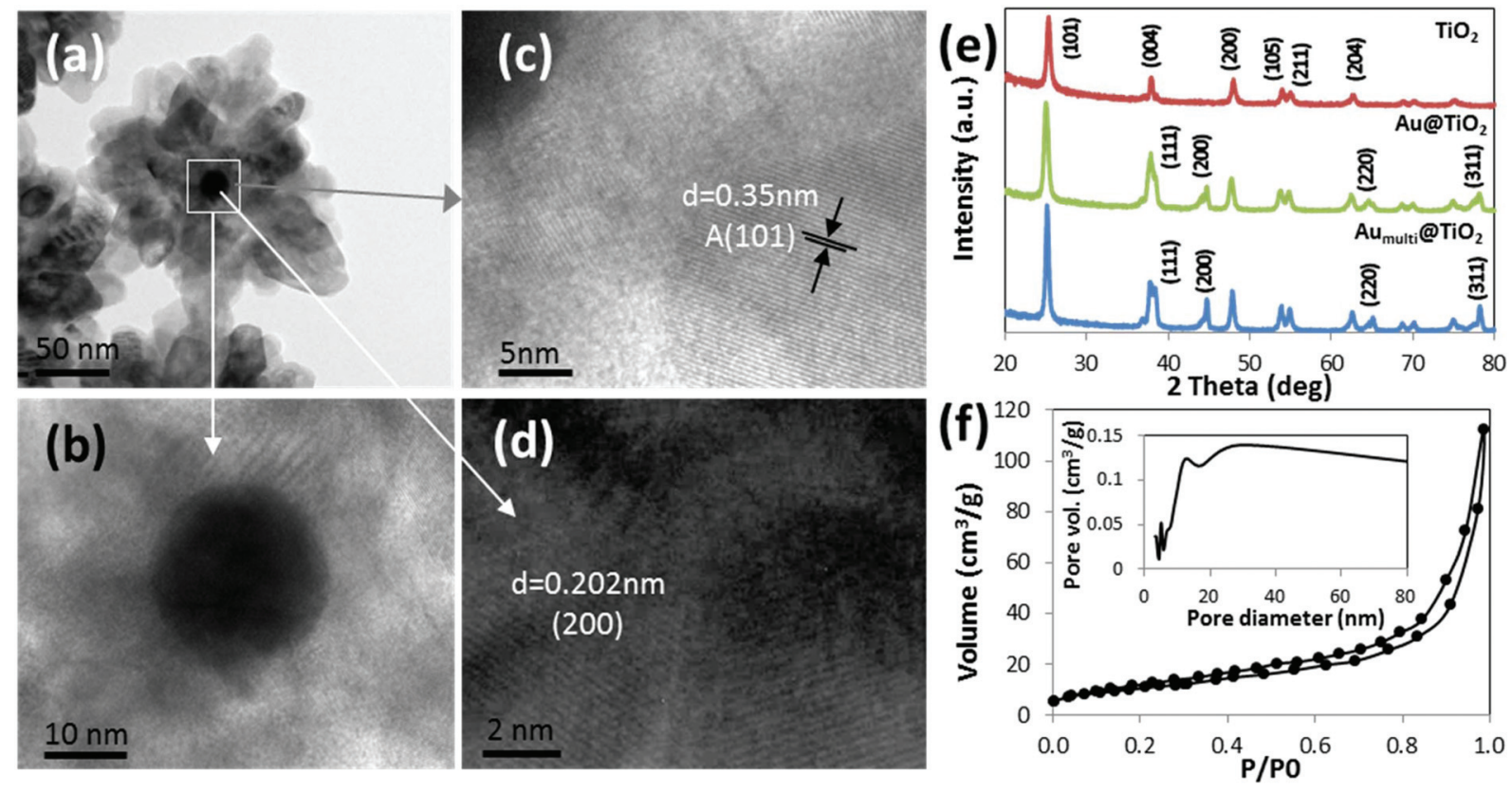

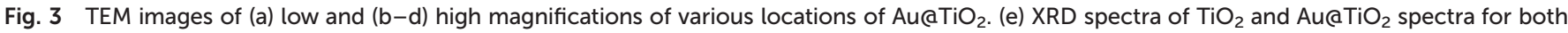
single and multiple cores. (f) $\mathrm{N}_{2}$ adsorption-desorption isotherm and the pore size distribution curves of single core $\mathrm{AuCTiO}_{2}$ (inset). 

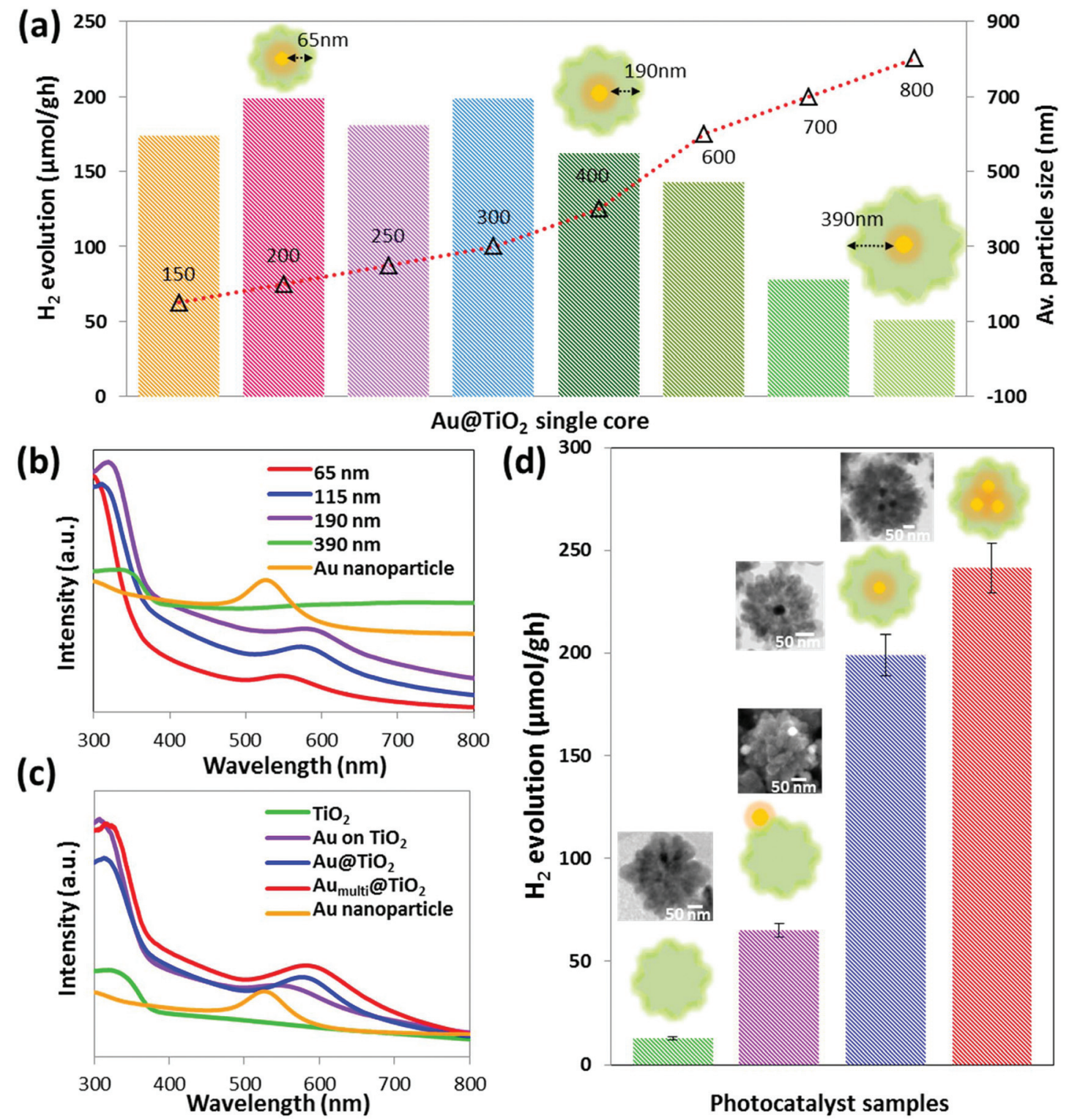

Fig. 4 (a) Photocatalytic $\mathrm{H}_{2}$ evolution and (b) absorption spectra of different shell thickness with fixed $20 \mathrm{~nm}$ Au core. (c) Absorption spectra and (d) $\mathrm{H}_{2}$ evolution of $\mathrm{TiO}_{2}$, Au on $\mathrm{TiO}_{2}$, single and multiple core Au@ $\mathrm{TiO}_{2}$ nanospheres.

(Fig. 4b) show two peaks whereby the weaker visible light absorption peaks are ascribed to the localized surface plasmon resonance (LSPR) response of Au nanoparticles. The physical origin of LSPR is the coherent oscillation of the conduction band electrons induced by the interacting electromagnetic field. A significant LSPR peak red-shift of $\mathrm{Au} @ \mathrm{TiO}_{2}$ nanospheres against pure Au nanoparticles (absorption wavelength of $\sim 525 \mathrm{~nm}$ ) is observed. This clearly reflects that the Au nanoparticles are surrounded by a porous shell of interconnected nanoparticles with a high refractive index. The existence of the oxide shell can be viewed to change the effective refractive index of the medium. Furthermore, the shell thickness is shown to affect the magnitude of the shift where peak shift of 23, 54 and $65 \mathrm{~nm}$ is observed with shell thickness of 65, 115 and $190 \mathrm{~nm}$, respectively. The magnitude of LSPR peaks red shift increase with shell thickness because the effective refractive index of the medium approaches the refractive index of the shell material. However, when the $\mathrm{TiO}_{2}$ shell thickness increases to $390 \mathrm{~nm}$, the light scattering becomes significant such that the weakening and complete masking of the LSPR peak is evident. ${ }^{17}$

Next, the effect of core content on light absorption and photocatalytic $\mathrm{H}_{2}$ generation is studied. The pure $\mathrm{TiO}_{2}$, single and multiple core samples were measured using UV-Vis spectroscopy at room temperature (Fig. 4c). The pure $\mathrm{TiO}_{2}$ sample shows an absorption peak solely in UV region because of its wide band gap. In contrast, the single and multiple core nanospheres display both UV and visible light absorption because of the presence of Au nanoparticles. In comparison, the multiple core samples exhibit a more intense and broader light 
absorption than the single core, both in UV and visible range. The increase in the intensity and broadness of the multiple core plasmon peak with respect to single core nanospheres may be associated to an increase in the Au core content and also an increase in the inter-particle electromagnetic coupling because of the confinement of the individual $\mathrm{Au}$ particles in close proximity. ${ }^{18}$ The UV-Vis absorption findings complement the photocatalytic performance because the rates of $\mathrm{H}_{2}$ production increase with the pure $\mathrm{TiO}_{2}$, single and multiple core nanospheres, corresponding to 13, 199 and $241 \mu \mathrm{mol} \mathrm{h} \mathrm{h}^{-1} \mathrm{~g}^{-1}$, respectively (Fig. 4d). The pure $\mathrm{TiO}_{2}$ nanospheres without $\mathrm{Au}$ core show approximately 15 times less $\mathrm{H}_{2}$ evolution than the $\mathrm{TiO}_{2}$ with spatially confined Au cores. This demonstrates the profound effect of the Au core on the photocatalytic activity. Here, the enhancement of the photocatalytic reactivity essentially arises from the unique electronic and optical interactions between the metallic cores and semiconducting shells over a length scale of a few tens of nanometer. Furthermore, it is observed that the modification of the core multiplicity by increasing the $\mathrm{Au}$ nanoparticles cores improves the photocatalytic properties. In essence, the probability of charge transfer and reduction of $\mathrm{H}^{+}$at the interface, as well as the photogenerated charge carriers that participate in photocatalytic reactions is increased. Finally, the effect of 'whereabout' of the Au nanoparticles, whether loaded on the surface or spatially confined within $\mathrm{TiO}_{2}$ matrix, is investigated. From the UV-Vis spectra (Fig. 4c), the LSPR peak of Au loaded on $\mathrm{TiO}_{2}$ surface is observed with a smaller shift against the pure $\mathrm{Au}$ spectrum. This is because of the fact that the Au nanoparticles are not embedded in the $\mathrm{TiO}_{2}$ shell. Hence, the refractive index of the surrounding Au medium does not change significantly. In addition, the intensity of the LSPR peak is weaker, which may suggest a weaker interaction between the $\mathrm{TiO}_{2}$ shell layer and the $\mathrm{Au}$ nanoparticles. Despite the greater exposure of the $\mathrm{Au}$ nanoparticles to water for the $\mathrm{Au}$ on $\mathrm{TiO}_{2}$ system, $\mathrm{H}_{2}$ production was still higher for the $\mathrm{Au@TiO}_{2}$ case. This strongly suggests that the surface contact area with water is not the only factor that determines the $\mathrm{H}_{2}$ production rate. As discussed later, electron trapping also plays a dominant role in the $\mathrm{H}_{2}$ generation. In the case of the $\mathrm{Au} @ \mathrm{TiO}_{2}$, the direct nucleation of $\mathrm{TiO}_{2}$ on the $\mathrm{Au}$ core results in a better interface between $\mathrm{Au}$ nanoparticles and $\mathrm{TiO}_{2}$ shell, while the geometric configuration allows optimal area for electronic interaction and also prevents the $\mathrm{Au}$ nanoparticles from dislodging. As a result, the rate of $\mathrm{H}_{2}$ production of the surface loaded $\mathrm{Au}$ nanoparticles yields $65 \mu \mathrm{mol} \mathrm{h} \mathrm{h}^{-1} \mathrm{~g}^{-1} \mathrm{H}_{2}$, which is $3-4$ times less than the spatially confined $\mathrm{Au}$ nanoparticles. These findings highlight that while it is important to incorporate $\mathrm{Au}$ metal to $\mathrm{TiO}_{2}$ for higher photocatalytic performance through enhanced light absorption and charge transfer, the "whereabout" of the Au nanoparticles affects the photocatalytic properties.

In order to understand the carrier dynamics in metal nanoparticle and metal oxide system, time-resolved absorption was measured. The charge transfer between $\mathrm{TiO}_{2}$ and Au nanoparticle was probed by monitoring the transient absorption decay at $680 \mathrm{~nm}$ following $350 \mathrm{~nm}$ laser pulse excitation of the
$\mathrm{Au} @ \mathrm{TiO}_{2}$ nanospheres as shown in Fig. 5a. The band gap excitation of $\mathrm{TiO}_{2}$ using UV-laser pulse results in charge separation followed by charge recombination and charge-trapping processes. The decay of the transmittance in the picosecond time scale represents the fraction of the electrons that are lost in the recombination process. ${ }^{19}$ The transmission-time profiles indicate a faster decay in $\mathrm{Au} @ \mathrm{TiO}_{2}$ nanospheres compares with pure $\mathrm{TiO}_{2}$ system. Therefore, it is obvious that the radiative charge recombination in $\mathrm{TiO}_{2}$ is substantially suppressed by $\mathrm{Au} @ \mathrm{TiO}_{2}$ nanospheres. This quenching phenomenon has been attributed to the effective trapping of photogenerated electron by $\mathrm{Au}$ nanoparticles. Furthermore, study on the effect of charge transfer on the photocatalytic hydrogen production performance of the four different designs of $\mathrm{TiO}_{2}$ nanospheres synthesized with/without $\mathrm{Au}$ nanoparticles, as well as surface
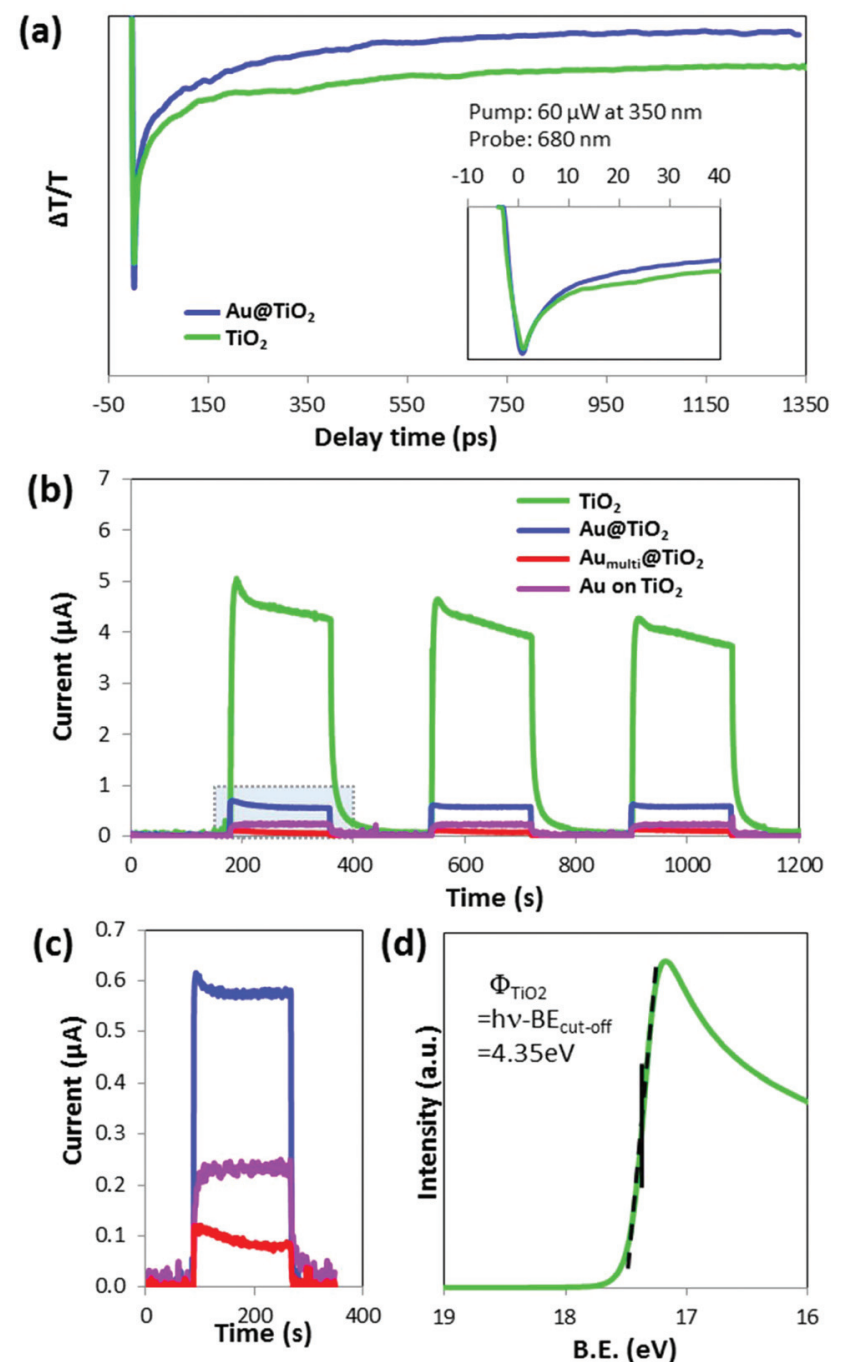

Fig. 5 (a) Transient absorption decay spectra of $350 \mathrm{~nm}$ laser pulse excitation of $\mathrm{TiO}_{2}$ and $\mathrm{Au} \mathrm{aTiO}_{2}$ nanospheres. (b) Photocurrent response of $\mathrm{TiO}_{2}$ nanospheres, $\mathrm{Au}$ on $\mathrm{TiO}_{2}$ and $\mathrm{Au} \mathrm{TTiO}_{2}$ (single and multiple core) electrodes under UV-Visible irradiation. (c) Magnified plot of (b) at the marked out region. (d) UPS spectra of $\mathrm{TiO}_{2}$ nanospheres in the valence band region. 
loaded or spatially confined Au nanoparticles was carried out using photoelectrochemical (PEC) measurements. Fig. 5b shows the photocurrent of pure $\mathrm{TiO}_{2}$ nanospheres $(\sim 4.25 \mu \mathrm{A})$ of almost five times higher than all the $\mathrm{TiO}_{2}$ with $\mathrm{Au}$ nanoparticles systems. The PEC set-up measures the photocurrent by collecting the photogenerated electrons through the photoanode. Upon UV light radiation, a fraction of the photogenerated electrons are transferred to the Au nanoparticles instead of transporting entirely to the transparent electrode and then through the external circuit, thus a lower photocurrent is obtained. Fig. 5c shows the magnified PEC plots of Fig. 5b at the indicated area. The single core $\mathrm{Au} @ \mathrm{TiO}_{2}$ system shows a higher photocurrent $(\sim 0.57 \mu \mathrm{A})$ compares to $\mathrm{Au}$ on $\mathrm{TiO}_{2}$

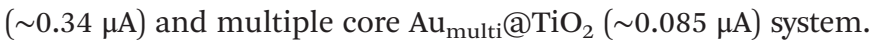
In regard to the spatially confined $\mathrm{Au}$ nanoparticles within $\mathrm{TiO}_{2}$ shell matrix, the photogenerated electrons are trapped within the $\mathrm{Au}$ nanoparticles such that the charge trapping probability increases with the Au nanoparticles content. Hence, the multiple cores $\mathrm{Au}_{\text {multi } @ \mathrm{TiO}_{2}}$ has lower photogenerated electrons reaching the cathode than the single core $\mathrm{Au} @ \mathrm{TiO}_{2}$. In the case of multiple $\mathrm{Au}$ nanoparticles that are loaded on $\mathrm{TiO}_{2}$ nanospheres, a higher photocurrent is measured as compared to multiple cores $\mathrm{Au}_{\text {multi }} @ \mathrm{TiO}_{2}$ because electrons trapped in Au nanoparticles, which are loaded on the surface of the $\mathrm{TiO}_{2}$ nanospheres may be transferred to the transparent electrode, thus contribute to the photocurrent. Moreover, the contact area of the multiple $\mathrm{Au}$ nanoparticles confined within $\mathrm{TiO}_{2}$ matrices $\left(\mathrm{Au}_{\text {multi }} @ \mathrm{TiO}_{2}\right)$ is higher than that of the $\mathrm{Au}$ nanoparticles on $\mathrm{TiO}_{2}$, hence leading to more charge trapping. From the PEC results, the $\mathrm{Au} @ \mathrm{TiO}_{2}$ nanospheres are shown to exhibit superior charge trapping capability, which translates to the inhibition of charge recombination, hence resulted in higher photocatalytic performances.
The band position and band bending of $\mathrm{TiO}_{2}$ were obtained by UPS (Fig. 5d). In the UPS measurement, binding energy was set as 0 at the Fermi level (Fermi onset: $21.75 \mathrm{eV}$ ). The work function of $\mathrm{TiO}_{2}$ was calculated to be $4.35 \mathrm{eV}$ by using $\varphi_{\mathrm{w}}=h \nu$ - $\mathrm{BE}_{\text {cut-off }}$ where $h \nu$ is the corrected kinetic energy $(21.75 \mathrm{eV})$. Based on the information of the band position and band bending obtained, band diagram was drawn to explain this charge trapping effect of the Au nanoparticles (Fig. 6). Au has a higher work function than the $\mathrm{TiO}_{2}$ semiconductor. In this case, the work function of $\mathrm{Au}$ nanoparticles is estimated to be $5.6 \mathrm{eV}^{20}$ When $\mathrm{TiO}_{2}$ and $\mathrm{Au}$ nanoparticles come in contact, the Fermi levels of the $\mathrm{Au}$ nanoparticles and $\mathrm{TiO}_{2}$ align, causing the band bending of $\mathrm{TiO}_{2}$ and the formation of Schottky barrier. In the presence of UV-Visible irradiation (Fig. 6), the excited electrons in $\mathrm{TiO}_{2}$ move from the valence band into the conduction band of $\mathrm{TiO}_{2}$. These electrons in the conduction band of the $\mathrm{TiO}_{2}$ are available to perform water reduction, which generates hydrogen. However, electrons in the conduction band of $\mathrm{TiO}_{2}$ can also recombine with the holes in the valence band thus limiting hydrogen production. In the presence of the Au nanoparticles where Fermi level is located at an energy just below the conduction band of $\mathrm{TiO}_{2}$, the electron in the $\mathrm{TiO}_{2}$ conduction band can be effectively transferred to the Au nanoparticles. ${ }^{21}$ At the semiconductorwater interface, the energy bands of an n-type semiconductor are bent upwards, the corresponding electrical field across the space charge layer drives holes in the valence band of $\mathrm{TiO}_{2}$ to the surface where the oxidation of sacrificial reagent occurs. Hence, the respective electric fields at both the interfaces enhance the separation of electron-hole pairs, thus reducing the recombination of photogenerated charges. Since the $\mathrm{Au} @ \mathrm{TiO}_{2}$ nanospheres are porous, good accessibility of water to $\mathrm{Au}$ interface allow hydrogen to be generated.

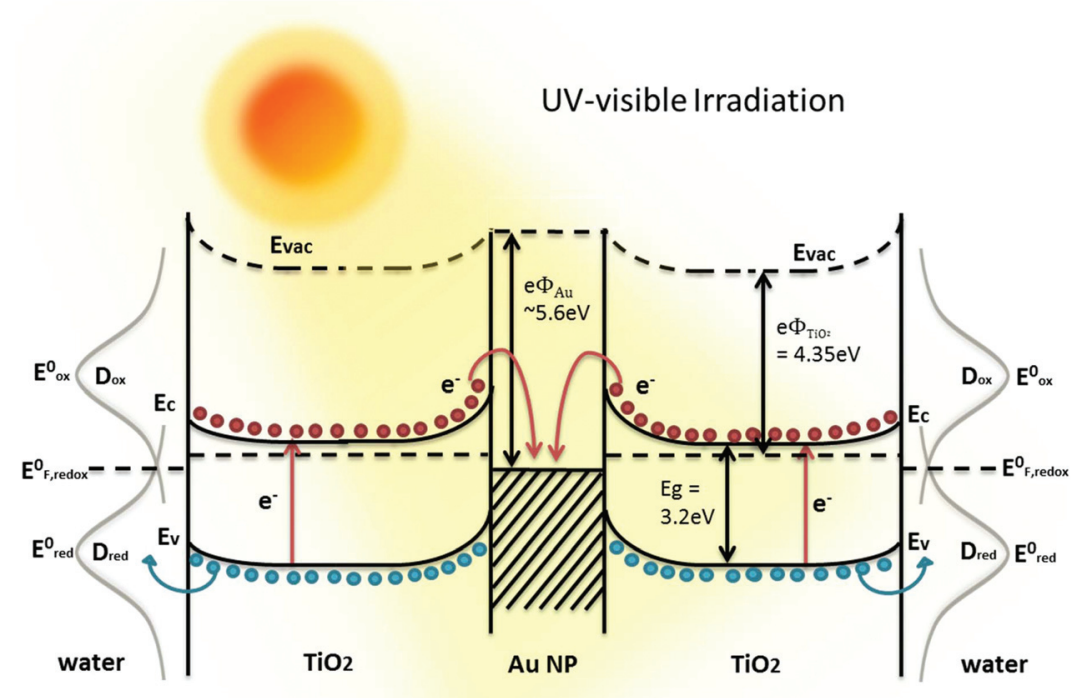

Fig. 6 Proposed photocatalytic mechanism and energy band diagram of the $\mathrm{Au@TiO}_{2}$ nanospheres under sunlight irradiation. 
The photocatalytic stability of three samples of $\mathrm{Au}$ on $\mathrm{TiO}_{2}$ and $\mathrm{Au} @ \mathrm{TiO}_{2}$ (single and multiple) core-shell nanospheres were tested through three cycles of two hours sacrificial water splitting experiment. The $\mathrm{Au}$ on $\mathrm{TiO}_{2}$ shows a decrease of $33 \%$ in $\mathrm{H}_{2}$ evolution, whereas the $\mathrm{Au} @ \mathrm{TiO}_{2}$ single and multiple core-shell nanospheres exhibit $\pm 5 \%$ variation in $\mathrm{H}_{2}$ evolution (Fig. 7a). Moreover, it is noted that when UV, as well as both UV-visible light are irradiated onto the $\mathrm{Au}_{\text {multi }} @ \mathrm{TiO}_{2}$ and $\mathrm{TiO}_{2}$ samples, no increase in the rate of hydrogen production is

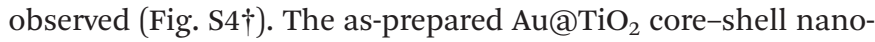
spheres are evidently more stable photocatalyst than $\mathrm{Au}$ on $\mathrm{TiO}_{2}$. In order to investigate the origin of prolonged catalytic activity of the spatially confined metal cores nanospheres as opposed to conventional surface loaded metal particles, The XPS characterization is carried out on the samples after the photocatalytic cycling experiments (Fig. 7b). The Ti2p spectra reveal two peaks, Ti2p $1 / 2$ and Ti2p $3 / 2$ with an energy splitting of $5.7 \mathrm{eV}$. This confirms the existence of titanium as $\mathrm{Ti}^{4+}$. The Ti2p 3/2 binding energies of $\mathrm{Au}$ on $\mathrm{TiO}_{2}$ and $\mathrm{Au} @ \mathrm{TiO}_{2}$ (both single and multiple cores) are at 458.3 and $458.6 \mathrm{eV}$, respectively. The approximate $0.3 \mathrm{eV}$ shift can be attributed to the transfer of $\mathrm{TiO}_{2}$ conduction band electron to Au nanoparticles resulting in a decrease in the outer electron cloud density of the Ti ions. ${ }^{16}$ This implies a stronger interaction between $\mathrm{TiO}_{2}$ and $\mathrm{Au}$ nanoparticles when they are spatially confined with a core-shell structure. ${ }^{16,22,23}$ The Ti2p $3 / 2$ peaks at 458.3-458.6 eV correspond to titanium in the anatase phase, which agrees well with the XRD and TEM structural analyses. The $\mathrm{O}$ 1s spectrum of $\mathrm{Au}$ on $\mathrm{TiO}_{2}$ exhibits two peaks at 529.3 and $533.0 \mathrm{eV}$. The main peak at $529.3 \mathrm{eV}$ is attributed to the oxygen bound to the tetravalent Ti ions, while the shoulder peak is attributed to the adsorbed hydroxyl groups (Ti-OH), defective oxides and/or adsorbed water. It is observed that O1s

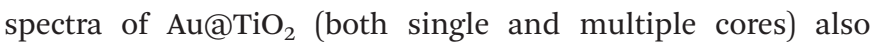
exhibit a positive shift (approximately $0.6 \mathrm{eV}$ ) relative to the $\mathrm{Au}$ on $\mathrm{TiO}_{2}$. Similarly, it has been reported that an increase in binding energies for both Ti2p and O1s can be attributed to strong interaction between $\mathrm{Au}$ and $\mathrm{TiO}_{2}{ }^{21}$ Such strong electronic interaction is beneficial to photocatalysis because it is expected to facilitate charge transfer-separation at the inter-
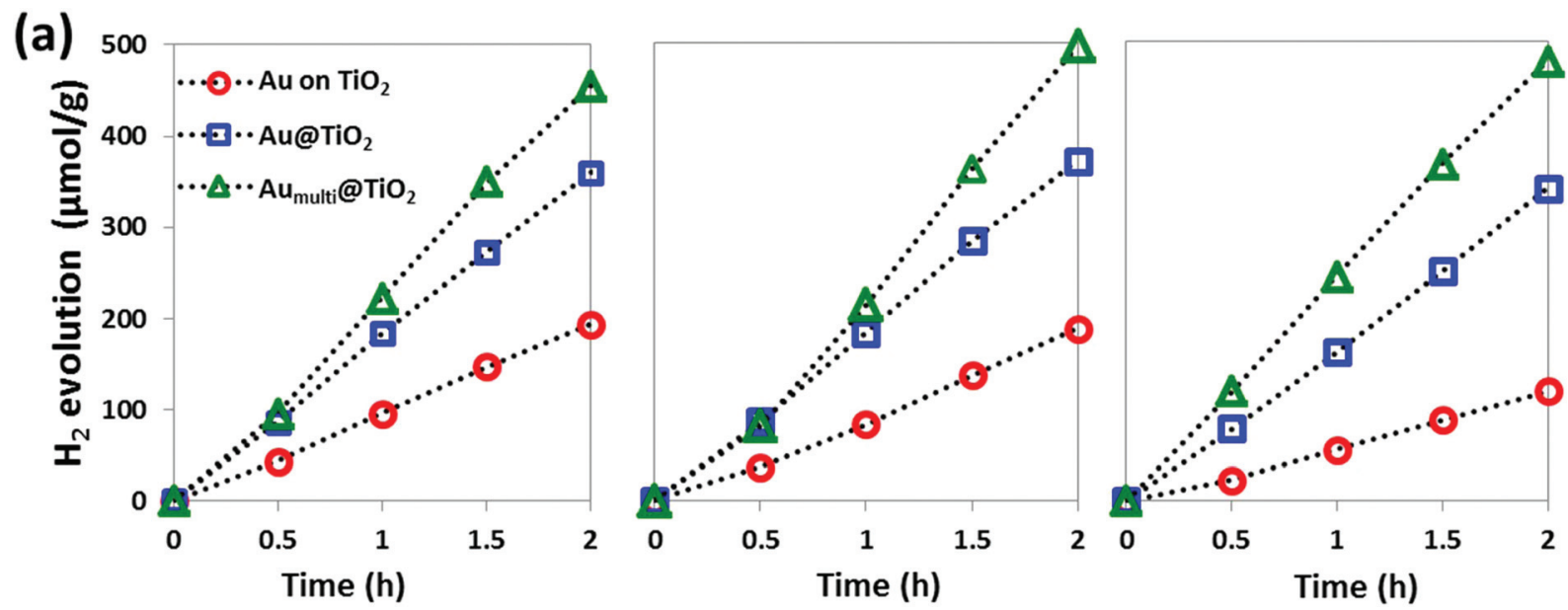

(b)
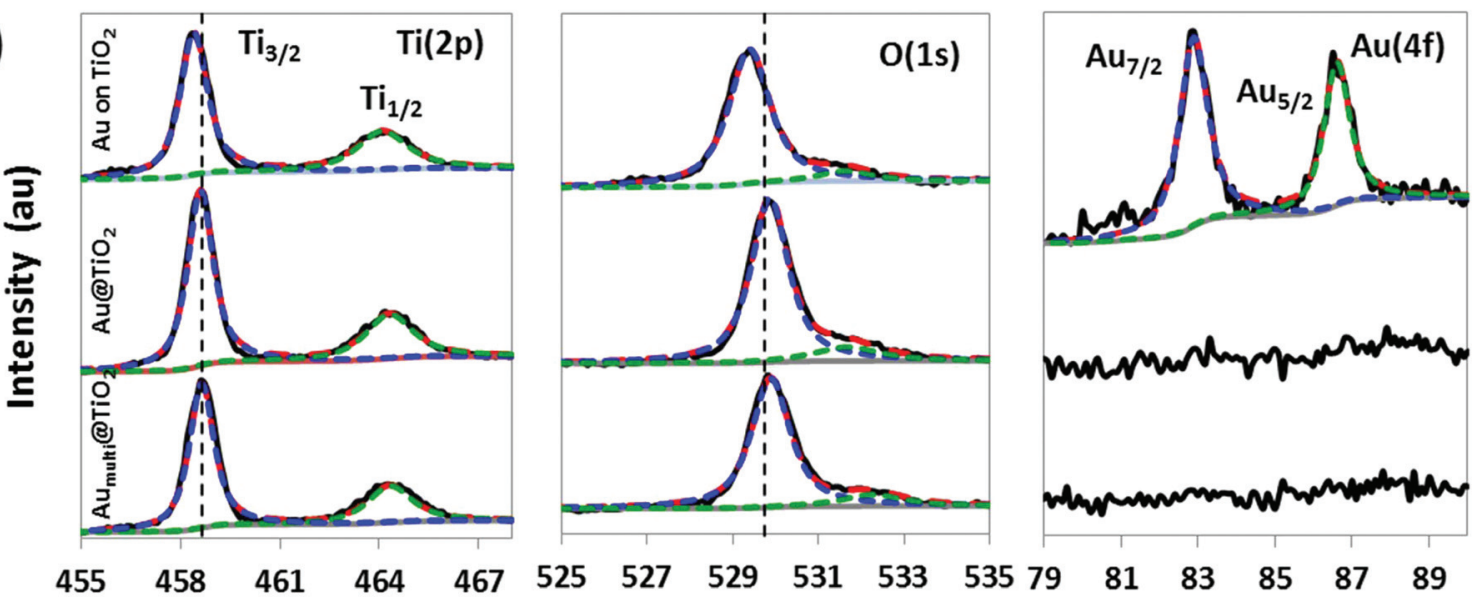

Binding Energy (eV)

Fig. 7 (a) Photocatalytic $\mathrm{H}_{2}$ evolution cycles of $\mathrm{Au}$ on $\mathrm{TiO}_{2}$ and $\mathrm{AuQTiO}$ (single and multiple) core-shell nanospheres. (b) XPS spectra of the various samples after the photocatalytic cycling experiments. 
face. Hence, the sacrificial water splitting of spatially confined $\mathrm{Au}$ cores is shown to yield higher $\mathrm{H}_{2}$ compared to the surface loaded $\mathrm{Au}$ nanoparticles. From the Au $4 \mathrm{f}$ spectra, it is noted that $\mathrm{Au}$ peaks are not detected for both the single and multiple cores Au@ $\mathrm{TiO}_{2}$ samples because the Au nanoparticles are confined within the shell thickness of $\sim 90 \mathrm{~nm}$. This also verifies the homogeneity of $\mathrm{Au}$ nanoparticles embedded in $\mathrm{TiO}_{2}$ matrix. In contrast, Au signal is detected on the $\mathrm{Au}$ on $\mathrm{TiO}_{2}$ sample, where the binding energy of spin-orbit split Au4f $\mathrm{f}_{7 / 2}$ is $82.9 \mathrm{eV}$ and $\mathrm{Au} 4 \mathrm{f}_{5 / 2}$ is $86.6 \mathrm{eV}$, separated by $3.7 \mathrm{eV}$. This indicates that the $\mathrm{Au}$ nanoparticles are present in metallic state on the surface of $\mathrm{TiO}_{2}$ nanospheres. Hence, it can be inferred that the instability of the catalytic activity of the conventional surface loaded Au nanoparticles is not because of the oxidation of metal particles, but more likely because of the dislodging/leaching of $\mathrm{Au}$ nanoparticles, which are loosely attached to the $\mathrm{TiO}_{2}$ surface. In this regard, it is worth pointing out that the confinement of the $\mathrm{Au}$ nanoparticles within $\mathrm{TiO}_{2}$ matrix not only boosts the charge transfer because of the strong electronic interaction between the core and shell, but also to alleviate $\mathrm{Au}$ nanoparticles against leaching. Therefore, another compelling reason to spatially confine the metal nanoparticles with core-shell structure is to ensure prolonged and stable photocatalytic activity.

\section{Conclusions}

We demonstrated a facile aqueous method of confining metal nanoparticles within shell matrix via in situ encapsulation without the use of template or multiple/post processes. The synthesis route is highly controllable such that the fine structural tuning of core and shell components can offer various model systems for photocatalytic studies. Moreover, we have proven that $\mathrm{Au}$ nanoparticles when introduced as cores rather than on the surface, as well as in abundance can dramatically influence the overall photocatalytic performance. We have also demonstrated the effectiveness of multiple $\mathrm{Au}$ nanoparticles embedded as core functions as charge accumulator to reduce the recombination of electron-hole pairs. The rational design of novel catalyst has fulfilled various functions, including enhanced light absorption, improved electron-hole separation, accessible redox reactions and stable/prolonged catalytic activity. This work is of fundamental relevance to the design of stable and efficient photocatalysts for sustainable energy and environmental applications.

\section{Experimental section}

\section{Synthesis of $\mathrm{TiO}_{2}$ nanospheres}

The different amounts of $0.04 \mathrm{M}$ TiF4 stock solutions ranging from $3 \mathrm{~mL}$ to $18 \mathrm{~mL}$ were diluted to $100 \mathrm{~mL}$ with deionized water and stirred for $10 \mathrm{~min}$. The solutions were transferred to Teflon-lined stainless steel autoclaves and heated in a binder oven at $180{ }^{\circ} \mathrm{C}$ for $3 \mathrm{~h}$. After that, the products were cooled to room temperature and separated by centrifuge at $4000 \mathrm{rpm}$ for 5 min, then washed with distilled water. The cycles of separation and washing were repeated three times to remove the remaining ions. The final samples were dried in a $90{ }^{\circ} \mathrm{C}$ oven and collected for further use.

\section{Synthesis of Au nanoparticles}

$4 \mathrm{~mL}$ of $0.01 \mathrm{M}$ sodium citrate solution was added to $12 \mathrm{~mL}$ of $0.01 \mathrm{M} \mathrm{HAuCl}_{4}$ solution. The mixture was stirred vigorously for $5 \mathrm{~min}$. Then, $3 \mathrm{~mL}$ of $0.01 \mathrm{M}$ ascorbic acid was added dropwise to the mixture while stirring. The mixture turned to orchid colour and then rapidly to wine red colour, which indicated gold nanoparticles had been formed.

\section{Synthesis of $\mathrm{Au@TiO}$, core-shell}

The as-prepared gold nanoparticle solution was stirred continuously for another $10 \mathrm{~min}$. Then, different amounts of $0.04 \mathrm{M}$ $\mathrm{TiF}_{4}$ stock solutions, ranging from $3 \mathrm{~mL}$ to $18 \mathrm{~mL}$ were added to the Au nanoparticle solution. The mixture was subsequently diluted to $100 \mathrm{~mL}$ with deionized water and transferred to Teflon-lined stainless steel autoclaves. The condition of the hydrothermal reaction and washing process is the same as mentioned above for the synthesis of $\mathrm{TiO}_{2}$ nanospheres. The yield of $\mathrm{Au}-\mathrm{TiO}_{2}$ heterostructures was calculated to be $82.3 \%$ after growth and washing.

\section{Synthesis of $\mathrm{Au}$ on $\mathrm{TiO}_{2}$ structure}

The as-prepared Au nanoparticles solution was added into $20 \mathrm{mg}$ of as-prepared $\mathrm{TiO}_{2}$ nanospheres. The mixture solution was stirred for $12 \mathrm{~h}$. The sample was rinsed with DI water and then centrifuged. The final samples were dried in a $90{ }^{\circ} \mathrm{C}$ oven and collected for further use.

\section{Materials characterization}

The scanning electron microscopy (SEM) characterization was carried out using a JEOL FEG JSM 6700F field-emission operating at $15 \mathrm{kV}$. X-ray photoelectron spectroscopy (XPS), VG ESCALAB 220I-XL system equipped with an $\mathrm{Mg} \mathrm{K} \alpha$ X-ray source was employed chemical for composition studies. The crystalline structures were analyzed using transmission electron microscopy (TEM, Phillips FEG CM300) operated at $200 \mathrm{kV}$ and X-ray diffraction (XRD, Philips X-ray diffractometer equipped with graphite-monochromated $\mathrm{Cu} \mathrm{K} \alpha$ radiation at $\lambda=1.541 \AA$ ). Absorption spectra were obtained using a Shimadzu UV-3600 UV-vis spectrophotometer. BrunauerEmmett-Teller (BET) measurements were conducted using Quantachrome Nova 1200 with $\mathrm{N}_{2}$ as the adsorbate at liquid nitrogen temperature. Photoelectrochemical (PEC) samples were prepared on fluorine doped tin oxide (FTO) substrates (QZ hybrid supplies, <10 $\Omega$ per sq) using a doctor blade method. Slurries were prepared from the various nanostructure powders and deionised water, which were then applied on the surface of the FTO glass using a glass rod. The coated FTO glass substrates were dried at $70{ }^{\circ} \mathrm{C}$ on a hot plate. The PEC measurements were carried out with a potentiostat in a 2 electrode configuration, using the photoanode prepared on FTO 
and a Pt counter electrode with $0 \mathrm{~V}$ bias in $0.1 \mathrm{M} \mathrm{Na}_{2} \mathrm{SO}_{4}$ electrolyte. The ultraviolet photoelectron spectroscopy (UPS) was performed with a He I (21.21 eV) radiation (VG Scientific ESCA Lab Mark 2). The binding energy of all spectra was calibrated using a gold $\mathrm{Au}$ ) metal, at which the Fermi onset is located at kinetic energy of $21.75 \mathrm{eV}$. The $\mathrm{H}_{2}$ evolution measurements were carried out using $30 \mathrm{mg}$ of photocatalyst and $10 \mathrm{~mL}$ DI water $(10 \%$ methanol) contained in a quartz vial and illuminated with a $300 \mathrm{~W}$ Xe lamp (Excelitas, PE300BFM). The wavelength range and light intensity of the Xe lamp is

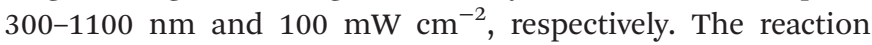
mixture was purged with Ar gas for 15 min prior to measurements. The reaction mixture was syringe drawn $(100 \mu \mathrm{l})$ to sample the gas composition using gas chromatographer (Shimadzu, GC-2014AT).

\section{Acknowledgements}

This work is supported by the A*Star grant R-263-000-A96-305.

\section{References}

1 D. Djoumessi Lekeufack, A. Brioude, A. Mouti, J. G. Alauzun, P. Stadelmann, A. W. Coleman and P. Miele, Core-shell $\mathrm{Au} @\left(\mathrm{TiO}_{2}, \mathrm{SiO}_{2}\right)$ nanoparticles with tunable morphology, Chem. Commun., 2010, 46, 4544-4546.

2 G. Oldfield, T. Ung and P. Mulvaney, Au@ $\mathrm{SnO}_{2}$ Core-Shell Nanocapacitors, Adv. Mater., 2000, 12, 1519-1522.

3 T. Hirakawa and P. V. Kamat, Charge Separation and Catalytic Activity of $\mathrm{Ag} @ \mathrm{TiO}_{2}$ Core-Shell Composite Clusters under UV-Irradiation, J. Am. Chem. Soc., 2005, 127, 39283934.

4 C. Z. Wu, Z. Y. Lim, C. Zhou, W. G. Wang, S. Zhou, H. Yin and Y. Zhu, A soft-templated method to synthesize sintering resistant Au-mesoporous-silica core-shell nanocatalysts with sub-5 nm single-cores, Chem. Commun., 2013, 49, 3215-3217.

$5 \mathrm{~J}$. H. Byeon and Y. W. Kim, $\mathrm{Au}-\mathrm{TiO}_{2}$ Nanoscale Heterodimers Synthesis from an Ambient Spark Discharge for Efficient Photocatalytic and Photothermal Activity, ACS Appl. Mater. Interfaces, 2014, 6, 763-767.

6 J. Lu, B. Fu, M. C. Kung, G. Xiao, J. W. Elam, H. H. Kung and P. C. Stair, Coking and sintering resistant palladium catalysts achieved through atomic layer deposition, Science, 2012, 335, 1205-1208.

7 S. Ikeda, S. Ishino, T. Harada, N. Okamoto, T. Sakata, H. Mori, S. Kuwabata, T. Torimoto and M. Matsumura, Ligand-Free Platinum Nanoparticles Encapsulated in a Hollow Porous Carbon Shell as a Highly Active Heterogeneous Hydrogenation Catalyst, Angew. Chem., Int. Ed., 2006, 45, 7063-7066.

8 I. Lee, Q. Zhang, J. Ge, Y. Yin and F. Zaera, Encapsulation of Supported Pt Nanoparticles with Mesoporous Silica for Increased Catalyst Stability, Nano Res., 2011, 4, 115-123.
9 J. Ge, Q. Zhang, T. Zhang and Y. Yin, Core-Satellite Nanocomposite Catalysts Protected by a Porous Silica Shell: Controllable Reactivity, High Stability, and Magnetic Recyclability, Angew. Chem., Int. Ed., 2008, 47, 8924-8928.

10 X. Z. Kong, W. Jiang, X. Jiang and X. Zhu, Preparation of core-shell and hollow polyurea microspheres via precipitation polymerization using polyamine as crosslinker monomer, Polym. Chem., 2013, 4, 5776-5784.

11 H. Dong, M. Zhu, J. A. Yoon, H. Gao, R. Jin and K. Matyjaszewski, One-Pot Synthesis of Robust Core/Shell Gold Nanoparticles, J. Am. Chem. Soc., 2008, 130, 1285212853.

12 H. G. Yang, C. H. Sun, S. Z. Qiao, J. Zou, G. Liu, S. C. Smith, H. M. Cheng and G. Q. Lu, Anatase $\mathrm{TiO}_{2}$ single crystals with a large percentage of reactive facets, Nature, 2008, 453, 638-641.

13 R. Menzel, A. Duerrbeck, E. Liberti, H. C. Yau, D. McComb and M. S. P. Shaffer, Determining the Morphology and Photocatalytic Activity of Two-Dimensional Anatase Nanoplatelets Using Reagent Stoichiometry, Chem. Mater., 2013, 25, 2137-2145.

14 X. F. Wu, H. Y. Song, J. M. Yoon, Y. T. Yu and Y. F. Chen,

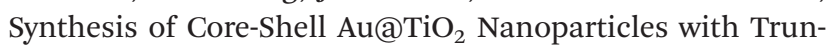
cated Wedge-Shaped Morphology and Their Photocatalytic Properties, Langmuir, 2009, 25, 6438-6447.

15 Y. Konishi, I. Tanabe and T. Tatsuma, Stable spectral dip formation and multicolour changes of plasmonic gold nanoparticles on $\mathrm{TiO}_{2}$, Chem. Commun., 2013, 49, 606-608.

16 Y. Liu, L. F. Chen, J. C. Hu, J. L. Li and R. Richards, $\mathrm{TiO}_{2}$ Nanoflakes Modified with Gold Nanoparticles as Photocatalysts with High Activity and Durability under near UV Irradiation, J. Phys. Chem. C, 2010, 114, 1641-1645.

17 P. Ramasamy, D. M. Seo, S. H. Kim and J. W. Kim, Effects of $\mathrm{TiO}_{2}$ shells on optical and thermal properties of silver nanowires, J. Mater. Chem., 2012, 22, 11651-11657.

18 S. K. Ghosh and T. Pal, Interparticle Coupling Effect on the Surface Plasmon Resonance of Gold Nanoparticles: From Theory to Applications, Chem. Rev., 2007, 107, 4797-4862.

19 V. Subramanian, E. E. Wolf and P. V. Kamat, Catalysis with $\mathrm{TiO}_{2} /$ Gold Nanocomposites. Effect of Metal Particle Size on the Fermi Level Equilibration, J. Am. Chem. Soc., 2004, 126, 4943-4950.

20 N. T. Khoa, S. W. Kim, D. H. Yoo, E. J. Kim and S. H. Hahn, Size-dependent work function and catalytic performance of gold nanoparticles decorated graphene oxide sheets, Appl. Catal., A, 2014, 469, 159-164.

21 Z. Bian, T. Tachikawa, W. Kim, W. Choi and T. Majima, Superior electron transport and photocatalytic abilities of metal nanoparticle-loaded $\mathrm{TiO}_{2}$ superstructures, J. Phys. Chem. C, 2012, 116, 25444-25453.

22 D. Zhang, G. Li, F. Wang and J. C. Yu, Green synthesis of a self-assembled rutile mesocrystalline photocatalyst, CrystEngComm, 2010, 12, 1759-1763.

23 Y. Do, J. S. Choi, S. K. Kim and Y. Sohn, The interfacial nature of $\mathrm{TiO}_{2}$ and $\mathrm{ZnO}$ nanoparticles Modified by gold nanoparticles, Bull. Korean Chem. Soc., 2010, 31, 2170-2174. 